\title{
Reshaping anisotropic gold nanoparticles through oxidative etching: the role of the surfactant and nanoparticle surface curvature Haifeng Yuan, ${ }^{* a}$ Kris P. F. Janssen, ${ }^{a}$ Thomas Franklin, ${ }^{b}$ Gang Lu, ${ }^{a}$ Liang Su, ${ }^{a}$ Xian Gu ${ }^{b}$ Hiroshi Uji-i, ${ }^{a}$ Maarten B. J. Roeffaers, ${ }^{a, b}$ and Johan Hofkens ${ }^{* a, d}$
}

\author{
${ }_{5}$ Received (in $\left.X X X, X X X\right)$ Xth $X X X X X X X X X 20 X X$, Accepted Xth $X X X X X X X X X 20 X X$ \\ DOI: 10.1039/b000000x
}

We report an investigation on the effect of stabilization agents and surface curvatures on oxidative etching of three classes of anisotropically shaped gold nanoparticles namely, rods, 10 bipyramids and prisms. In particular, the dual role of the stabilizing agent CTAB in the etching process is explored, showing how it acts both as a source of bromine ions, accelerating etching and as a protection agent, resulting in anisotropic reshaping.

${ }_{15}$ Gold nanoparticles (GNPs) exhibit strong optical absorption/scattering at their surface plasmon resonances (SPRs) due to geometrical confinement of the collective electron oscillations (plasmons). By introducing geometric anisotropy, GNPs can exhibit multiple SPRs that correspond to the surface 20 plasmon modes along transversal and longitudinal directions. Particularly, the longitudinal SPRs (LSPRs), which strongly depend on the aspect ratio of GNPs ${ }^{1-3}$, are promising for sensing and optical applications because of their sensitivity to the surrounding medium ${ }^{4}$ and strong plasmon-related enhancements 25 of optical signals ${ }^{5,6}$.

Many applications such as plasmon-enhanced spectroscopy, however, require precisely defined LSPR wavelength to achieve optimal performance ${ }^{7-9}$. Therefore, it is important to control the dimensions and shape anisotropy of GNPs to obtain well-defined 30 LSPRs, tailored for specific applications. One straightforward way to achieve precise control is to vary the growth conditions during synthesis. Another approach is to reshape the synthesized nanoparticles by controlled etching ${ }^{10}$. Different from the former method, the latter one, which can be either isotropic or

35 anisotropic depending on the etchant used, provides very precise control over LSPRs so that they can be tuned almost continuously over a broad spectral range. Moreover, the controlled etching can help to produce nanoparticles that are difficult to synthesize, for instance, ultra smooth and uniform gold nanospheres ${ }^{11}$. Among 40 many etching processes, the oxidative etching of GNPs with hydrogen peroxide has drawn much attention because of its anisotropic nature of etching $2,10,12$. Therefore, the reshaping induced shift in LSPRs can also serve as a probe for chemical reactions ${ }^{13-15}$. Several studies have shown that the anisotropic 45 etching is linked to the capping agent and surface curvatures of $\mathrm{GNPs}^{2,12}$. However, the detailed role of CTABr as an often used capping agent is not yet answered ${ }^{16,17}$ and the reported surface
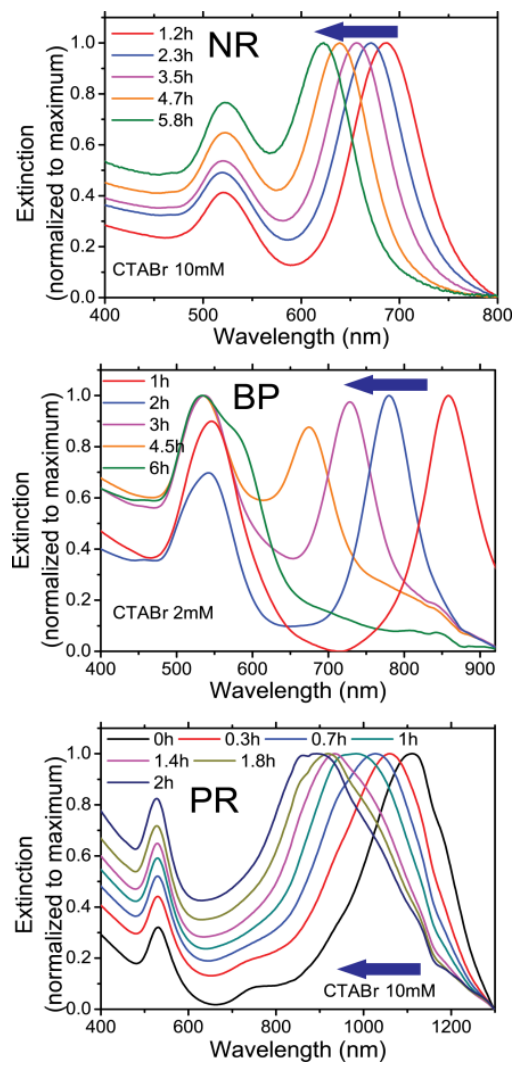

Fig. 1. Temporal evolution of the extinction spectra upon etching of NRs, BPs and PRs.

curvature effects remain puzzling ${ }^{2}$. Herein, we study the surfactant and shape dependent oxidative etching of anisotropic ${ }_{50}$ GNPs including nanorods $(\mathrm{NRs})^{18}$, bipyramids $(\mathrm{BPs})^{19}$ and prisms $(\mathrm{PRs})^{20}$.

Gold NRs $(42 \pm 4 \mathrm{~nm}$ by $13 \pm 3 \mathrm{~nm})$, BPs $(108 \pm 5 \mathrm{~nm}$ by $29 \pm 3 \mathrm{~nm})$ and PRs (120 $\pm 16 \mathrm{~nm}$ edge length, $8-10 \mathrm{~nm}$ thick) were synthesized using the reported seed-mediated methods ${ }^{18-20}$. In all 55 syntheses, we used cetyltrimethylammonium bromide $(\mathrm{CTABr})$ as capping agent. The anisotropic GNPs were then etched in solutions containing $30 \mathrm{mM} \mathrm{H} \mathrm{O}_{2}, 30 \mathrm{mM} \mathrm{HCl}$ and CTABr of different concentrations $(0.5 \mathrm{mM}, 1 \mathrm{mM}, 2 \mathrm{mM}, 5 \mathrm{mM}, 10 \mathrm{mM}$, $20 \mathrm{mM}$ and $50 \mathrm{mM})$ or $50 \mathrm{mM}$ cetyltrimethylammo60 nium chloride $(\mathrm{CTACl})$. Unless stated otherwise, the etchant 


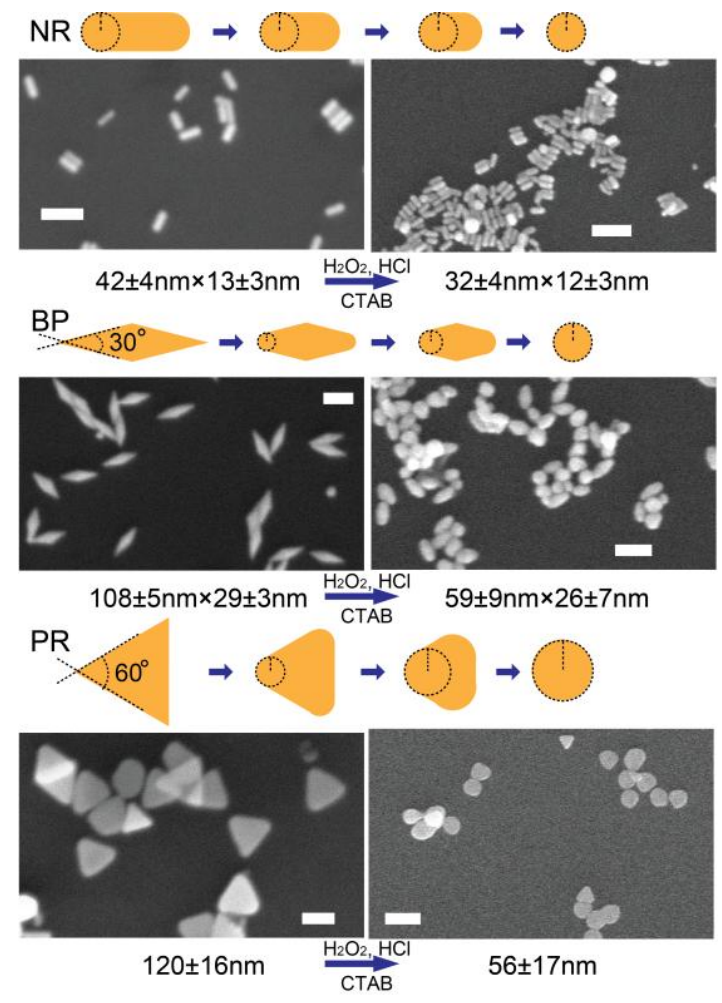

Fig. 2. A The schematic oxidative etching processes of NRs, BPs and PRs. SEM images recorded before and after etching for several hours demonstrate the reshaping process. The scale bars represent $100 \mathrm{~nm}$.

solutions were freshly prepared before use (details in ESI).

Upon addition of the etchant, continuous blue-shifts of the LSPRs were observed for all three kinds of GNPs (Fig. 1), implying reduced aspect ratios upon reshaping. The reshaping 5 was mainly shortening along longitudinal directions, as evident from SEM measurements shown in Fig. 2. Hereafter, we study the shortening by monitoring the evolution of the LSPR centroid wavelengths with time.

We first compare the etching rates of NRs under similar 10 etching condition but with different capping surfactants (CTABr and $\mathrm{CTACl}$ ). The blue-shifting LSPRs indicate shortening in both CTABr and CTACl stabilized samples, shown in Fig. 3a. The LSPR shift rate of CTABr stabilized NRs $(32 \mathrm{~nm} / \mathrm{h})$ is about 120 fold faster than that of CTACl stabilized NRs $(0.26 \mathrm{~nm} / \mathrm{h})$. We 15 notice that the only difference in both cases is the halide ions present in the capping agent. The greatly enhanced LSPR shift in presence of bromide ions clearly indicates the important role that bromide ions play during this $\mathrm{H}_{2} \mathrm{O}_{2}$ mediated etching procedure. Addition of bromide salts such as $\mathrm{KBr}$ to the $\mathrm{CTACl}$ etchant 20 solutions also markedly accelerates the etching process (Fig. S1).

We then further investigated the importance of bromide ions on the etching of NRs. After 24 hours aging, the $50 \mathrm{mM}$ CTABr etchant solution showed a color change from colorless to light yellow. An absorption peak at $265 \mathrm{~nm}$ and $390 \mathrm{~nm}$ can be then 25 observed in the UV-Vis spectra (see figure S2), indicative of $\mathrm{Br}_{3}{ }_{3}$ and $\mathrm{Br}_{2}$ formation respectively ${ }^{21}$. Moreover, $\mathrm{HBrO}$, which shall show very weak absorption around $260 \mathrm{~nm}^{21}$, can also generate from $\mathrm{Br}_{2}$ solution ${ }^{22}$ (figure S2). However, due to the strong absorption of $\mathrm{Br}_{3}{ }^{-}$at $265 \mathrm{~nm}$ which is very close to that of $\mathrm{HBrO}$
30 at $260 \mathrm{~nm}$, the $\mathrm{HBrO}$ absorption peak cannot be revealed in our experiment. To summarize, the reactions of bromide ions can be described with the following three formula ${ }^{22}$ :

$$
\begin{gathered}
2 \mathrm{Br}^{-}+\mathrm{H}_{2} \mathrm{O}_{2}+2 \mathrm{H}^{+} \rightarrow \mathrm{Br}_{2}+2 \mathrm{H}_{2} \mathrm{O} \\
3 \mathrm{Br}^{-}+\mathrm{H}_{2} \mathrm{O}_{2}+2 \mathrm{H}^{+} \rightarrow \mathrm{Br}_{3}^{-}+2 \mathrm{H}_{2} \mathrm{O} \\
\mathrm{Br}^{-}+\mathrm{H}_{2} \mathrm{O}_{2}+\mathrm{H}^{+} \rightarrow \mathrm{HBrO}+\mathrm{H}_{2} \mathrm{O}
\end{gathered}
$$
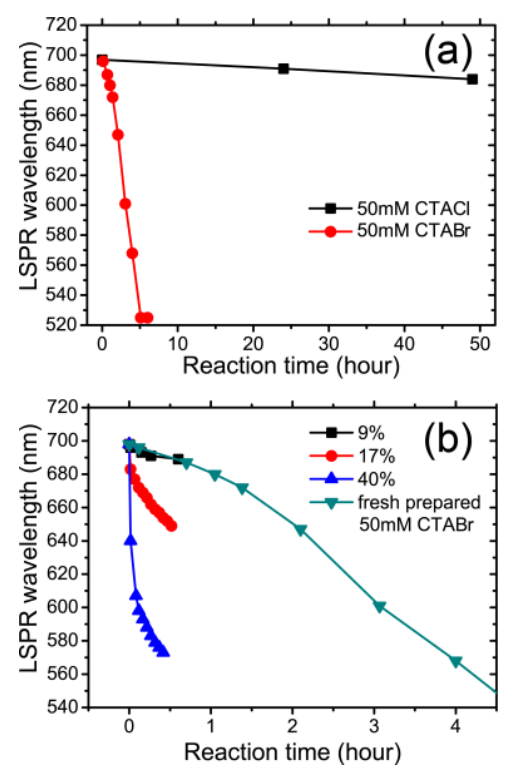

Fig. 3. Temporal evolution of the LSPR center positions of NRs. (a) Using CTACl (dark squares) or CTABr (red circles) as surfactant. (b) Etching on NRs using mixtures with 9\% (dark square), $17 \%$ (red circle) and $40 \%$ (blue up triangle) aged CTABr etchant (percentage in volume) and 50mM CTABr. Etching on NRs using freshly prepared etchant is shown in cyan, downward triangles.

In presence of $\mathrm{Br}_{2}$, the etching rate increased significantly; gold ${ }_{35} \mathrm{NRs}$ vanished almost instantly after being mixed with the aged etchant solution. To further demonstrate the effect of $\mathrm{Br}_{2}$ in aged solutions, we mixed the aged etchant solution with $50 \mathrm{mM}$ $\mathrm{CTABr}$ aqueous solution at different volume ratios. As shown in Fig. 3b, etching rate clearly increases upon increasing the volume ${ }_{40}$ percentages of the aged etchant solution. Even at a volume percentage as low as $9 \%$ for the aged solution, shortening of gold NRs is faster than with its freshly prepared counterpart, indicating that $\mathrm{Br}_{2}$ are very strong etchants for GNPs. Therefore, we can conclude that $\mathrm{Br}^{-}$is indeed promoting etching on gold NPs by ${ }_{45}$ providing extra oxidation channels other than direct oxidation by $\mathrm{H}_{2} \mathrm{O}_{2}$. To be exact, bromide ions promote the gold etching reaction by generating $\mathrm{Br}_{2}$ whose redox potentials is favourable.

Next to its role in promoting etching, CTABr also serves as capping agent to protect the surface of GNPs from etching. Thus, 50 the less protected tip region is more readily etched resulting in anisotropic reshaping. The protecting effect of the capping agent is counteracted by the promoting role of bromide ion on the etching speed, which implies that a higher CTABr concentration on the one hand will lead to faster etching but on the other hand ${ }_{55}$ will result in a better surface protection. At the highest $\mathrm{CTABr}$ concentration we observed faster spectral shifts in LSPR (Fig. 4). However, at lower concentrations a clear optimum between both effects was observed in the etching speed of NRs; at 

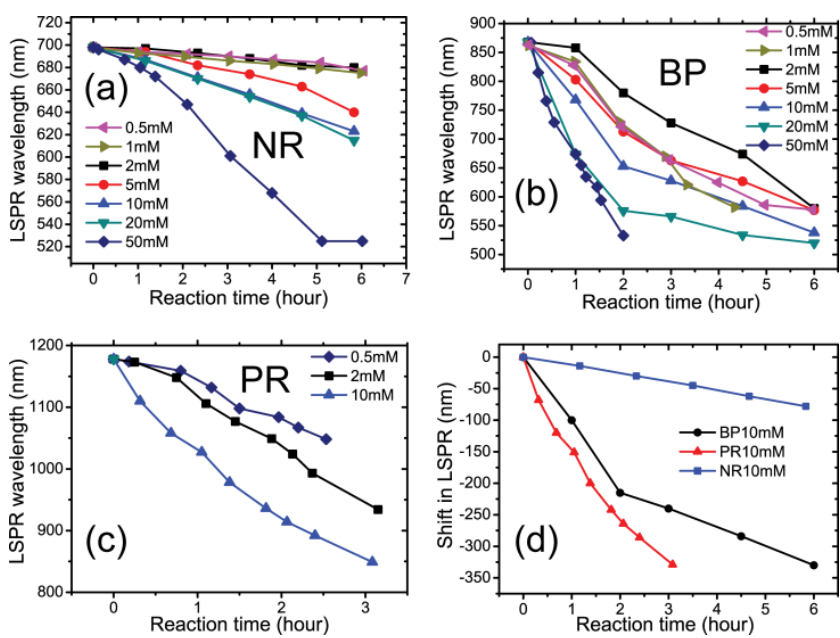

Fig. 4. LSPR evolution of different shaped NPs upon oxidative etching: (a) NRs, (b) BPs and (c) PRs. (d) LSPR shift from initial values of NRs, BPs and PRs under the same etching conditions.

concentrations of $0.5 \mathrm{mM}$ and $1 \mathrm{mM}$ LSPR shifts were comparable or even slightly faster than that at $2 \mathrm{mM} \mathrm{CTABr}$ concentration. Our finding implies much less capping protection at the tips of NRs at CTABr concentrations lower than $2 \mathrm{mM}$, 5 which agrees well with other reports ${ }^{4,23}$. Fig. 4 b shows a similar but more pronounced effect in BP samples, where the LSPR shift at $0.5 \mathrm{mM}$ and $1 \mathrm{mM} \mathrm{CTABr}$ concentrations are significantly faster than that at $2 \mathrm{mM} \mathrm{CTABr}$ concentration. In addition, gradually shortening along the transversal direction was clearly 10 observed on NRs and BPs at $0.5 \mathrm{mM} \mathrm{CTABr}$ concentration (see Table S1), indicating that the side walls of NRs and BPs were no longer fully covered by the capping agent at such a low $\mathrm{CTABr}$ concentration. Next to the aforementioned observations in solution, we found that etching became very heterogeneous for ${ }_{15}$ NRs on a solid substrate (Fig. S3). Analogously to etching at elevated temperatures ${ }^{12}$, such heterogeneous etching is likely due to the disturbed surface capping of GNPs on substrates. These results underscore the important role of surface capping in the etching process.

20 Next, we compare the reshaping of GNPs with different morphologies. At relatively low CTABr concentrations (0.5$2 \mathrm{mM}$ ), all three kinds of GNPs showed almost linear shift in LSPR with time, shown in Fig. 4. However, the LSPRs of GNPs showed different shifting behaviour at higher $\mathrm{CTABr}$ 25 concentrations. Different from NRs, which showed linear shift in LSPRs with time, BPs and PRs showed variations in LSPR shift rates with time. As demonstrated in Fig. 4b, LSPR shift rate in BPs was $110 \mathrm{~nm} / \mathrm{h}$ in the first two hours of etching of BPs and reduced to $26 \mathrm{~nm} / \mathrm{h}$ after four hours at $10 \mathrm{mM} \mathrm{CTABr}$ 30 concentration. Similarly, the LSPR shift rate in PRs was $151 \mathrm{~nm} / \mathrm{h}$ in the first hour of etching and decreased to $65 \mathrm{~nm} / \mathrm{h}$ after two hours under the same etching conditions, shown in Fig. 3c. Changes in LSPR shift rates with time observed in BPs and PRs are likely due to changes in tip surface curvatures upon 35 reshaping. Surface curvatures can be described using the equivalent radius $r$, as demonstrated in Fig. 2. In NRs, the surface curvature at tips remains the same during reshaping since the thickness of NRs remains constant. However, the tip surface curvatures in BPs and PRs evolve while reshaping, leading to an
40 increased $r$ thus a better coverage of surfactant on the tip surface. Therefore, the LSPR shift rates decrease as etching goes on.

In the following, we compare the LSPR shift rates of different GNPs in the first hour of reshaping. Fig. 4d clearly demonstrates that PRs showed the fastest LSPR shift and NRs showed the 45 slowest LSPR shift in the first hour of reaction under the same reaction condition. The LSPR shift rates of NRs, BPs and PRs in the first hour of etching at $10 \mathrm{mM} \mathrm{CTAB}$ concentration were $14 \mathrm{~nm} / \mathrm{h}, 110 \mathrm{~nm} / \mathrm{h}$ and $151 \mathrm{~nm} / \mathrm{h}$ respectively. However, the LSPR shift rate reflects the decrease in aspect ratio rather than 50 directly the shortening length. Therefore, the difference in their thickness has to be taken into account. To demonstrate the effect of GNP thickness, we applied the identical etching conditions (10 mM CTAB) to the NRs with different thickness $(13 \mathrm{~nm}$, $16 \mathrm{~nm}, 18 \mathrm{~nm}$ and $30 \mathrm{~nm}$ ). In general, LSPR shift rates of the 55 thicker NRs were found to be slower than that of the thinner NRs (see Fig. S3). For example, we observed LSPR shift rates of $6 \mathrm{~nm} / \mathrm{h}$ and $14 \mathrm{~nm} / \mathrm{h}$ for the NRs with thicknesses of $30 \mathrm{~nm}$ and $13 \mathrm{~nm}$ respectively. The ratio between LSPR shift rates and NRs' diameters scaled inversely $(14 / 6 \approx 30 / 13 \approx 2.3)$. Comparing 60 the NRs and BPs of similar thickness $(30 \mathrm{~nm})$, we found that the LSPR shift during the first hour in BPs $(110 \mathrm{~nm})$ was 18 times larger than that in NRs $(6 \mathrm{~nm})$. Assuming LSPRs of NRs and BPs follow similar dependence on the aspect ratios ${ }^{1,2,24}$, we estimate the longitudinal shortening length in BPs is more than 18 times 65 faster than that in NRs, which is in contradiction with previous report ${ }^{2}$, where different surfactants and experimental conditions were applied. Our observation is further supported by SEM micrographs on GNPs at different etching stages under identical conditions, where BPs showed about 15 times faster shortening 70 rates than NRs. For example, we found shortening of $3.3 \mathrm{~nm}$, $3.6 \mathrm{~nm}$ and $49.1 \mathrm{~nm}$ along the longitudinal axis in $13 \mathrm{~nm}$ thick $\mathrm{NRs}, 30 \mathrm{~nm}$ thick NRs and BPs respectively in the etching solution containing $0.5 \mathrm{mM} \mathrm{CTABr}$ during the first two hours (see table S1). PRs showed the fastest LSPR shift upon etching 75 due to two reasons. Firstly, PRs have sharply curved surfaces at the corners $\left(60^{\circ}\right)$, which makes the etching at the corners more efficient due to less capping. Secondly, due to PRs' finite thickness (8-10 nm), little changes in edge lengths will induce large changes in their aspect ratios thus large LSPR shift.

80 Besides the differences in surface curvatures of GNPs, their different crystal facets ${ }^{25}$, 26 , which may lead to different reactivities and surface binding, can also contribute to the overall differences. For instance, NRs possess $\{111\}\{001\}$ crystal facets at tips and $\{110\}$ facet at side walls ${ }^{25}$ whereas BPs have five $85\{111\}$ twinning planes ${ }^{26}$ and PRs have flat $\{111\}$ top surfaces and $\{112\}$ side surfaces ${ }^{27}$. However, since the anisotropic shapes of NPs are achieved by crystal growth with the help of surfactant binding ${ }^{20,26}$, these two factors are ultimately linked to each other and therefore are difficult to separate in this study.

\section{${ }_{90}$ Conclusions}

To summarize, we have applied oxidative etching to CTABr or CTACl stabilized NR, BP and PR samples. CTABr acts not only as the capping agents but also facilitates etching by generating $\mathrm{Br}_{2}$ upon oxidization by $\mathrm{H}_{2} \mathrm{O}_{2}$ under acid conditions. Serving as 95 the capping agent for NPs, CTABr protects NPs' surfaces against etching, which leads to strong curvature dependent anisotropic 
etching. We found that etching goes faster at sharp tips of GNPs, as evident from the faster etching on BPs compared with NRs. Moreover, the shortening speed relates to GNP's tip surface curvature, which can be deduced from reduced LSPR shift rates 5 of BPs and PRs with time. Our findings can guide future fabrication and reshaping of gold nanostructures for various physical and chemical applications including plasmon-enhanced spectroscopy and chemical sensing.

\section{${ }_{10}$ Notes and references}

This research received funding from the Research Foundation-Flanders (FWO, grant G.0990.11, G.0855.14, G.0197.11, G.0962.13, postdoctoral fellowship to H. Y., K. P. F. J. and G. L.), K.U. Leuven Research Fund (GOA2011/03, OT/12/059), the Flemish government through long term 15 structural funding Methusalem (Meth/08/04), the Hercules foundation (HER/08/021, HER/11/14), the Belgian Federal Science Policy Office (IAP-VI/27) and the European Research Council under the European Union's Seventh Framework Programme (FP7/2007-2013)/ERC Grant Agreement No. 291593 FLUOROCODE). M.B.J.R acknowledges the 20 European Research Council for financial support (ERC Starting Grant 307523). H. U acknowledges the European Research Council (ERC Starting Grant PLASMHACAT 280064) and the Japan Science and Technology Agency PRESTO program for financial support.

${ }_{25}{ }^{a}$ Department of Chemistry, KU Leuven, Celestijnenlaan 200F, B-3001 Leuven, Belgium.

${ }^{b}$ Department of Microbial and Molecular Systems, Centre for Surface Chemistry and Catalysis, KU Leuven, B-3001 Leuven, Belgium.

${ }^{c}$ PRESTO, Japan Science and Technology Agency (JST), 4-1-8 Honcho 30 Kawaguchi, Saitama 332-0012, Japan.

${ }^{d}$ Nano-Science Center, University of Copenhagen, Universitetsparken 5, 2100 Copenhagen, Denmark.

*To address correspondence: haifeng.yuan@chem.kuleuven.be; johan.hofkens@chem.kuleuven.be.

$35 \dagger$ Electronic Supplementary Information (ESI) available: [details of any supplementary information available should be included here]. See DOI: $10.1039 / \mathrm{b} 000000 \mathrm{x} /$

1. S. Link, M. B. Mohamed and M. A. El-Sayed, Journal of

$40 \quad$ Physical Chemistry B, 1999, 103, 3073-3077.

2. X. S. Kou, W. H. Ni, C. K. Tsung, K. Chan, H. Q. Lin, G. D. Stucky and J. F. Wang, Small, 2007, 3, 2103-2113.

3. A. Manjavacas and F. J. G. de Abajo, Nature Communications, 2014, 5, 7 .

$454 . \quad$ P. Zijlstra, P. M. R. Paulo and M. Orrit, Nature Nanotechnology, 2012, 7, 379-382.

5. H. Yuan, S. Khatua, P. Zijlstra, M. Yorulmaz and M. Orrit, Angewandte Chemie-International Edition, 2013, 52, 12171221.

506 A. M. Gabudean, M. Focsan and S. Astilean, Journal of Physical Chemistry C, 2012, 116, 12240-12249.

7. S. Khatua, P. M. R. Paulo, H. F. Yuan, A. Gupta, P. Zijlstra and M. Orrit, Acs Nano, 2014, 8, 4440-4449.

8. K. Munechika, Y. Chen, A. F. Tillack, A. P. Kulkarni, I. J. L.

55 Plante, A. M. Munro and D. S. Ginger, Nano Letters, 2010, 10, 2598-2603.

9. E. C. Le Ru and P. G. Etchegoin, in Annual Review of Physical Chemistry, Vol 63, eds. M. A. Johnson and T. J. Martinez, Annual Reviews, Palo Alto, 2012, vol. 63, pp. 65-87.

$6010 . \quad$ C. K. Tsung, X. S. Kou, Q. H. Shi, J. P. Zhang, M. H. Yeung, J. F. Wang and G. D. Stucky, Journal of the American Chemical Society, 2006, 128, 5352-5353.

11. Y. J. Lee, N. B. Schade, L. Sun, J. A. Fan, D. R. Bae, M. M. Mariscal, G. Lee, F. Capasso, S. Sacanna, V. N. Manoharan and G. R. Yi, Acs Nano, 2013, 7, 11064-11070.

65

$12 . \quad$ W. H. Ni, H. J. Ba, A. A. Lutich, F. Jackel and J. Feldmann, Nano Letters, 2012, 12, 4647-4650.
13. X. Liu, S. Y. Zhang, P. L. Tan, J. Zhou, Y. Huang, Z. Nie and S. Z. Yao, Chemical Communications, 2013, 49, 1856-1858.

$7014 . \quad$ J. M. Liu, L. Jiao, L. P. Lin, M. L. Cui, X. X. Wang, L. H. Zhang, Z. Y. Zheng and S. L. Jiang, Talanta, 2013, 117, 425430.

15. X. J. Yang, Y. B. Yu and Z. Q. Gao, Acs Nano, 2014, 8, 49024907.

$7516 . \quad$ T. Wen, H. Zhang, X. P. Tang, W. G. Chu, W. Q. Liu, Y. L. Ji, Z. J. Hu, S. Hou, X. N. Hu and X. C. Wu, Journal of Physical Chemistry C, 2013, 117, 25769-25777.

17. L. Saa, M. Coronado-Puchau, V. Pavlov and L. M. LizMarzan, Nanoscale, 2014, 6, 7405-7409.

8018 B. Nikoobakht and M. A. El-Sayed, Chem. Mat., 2003, 15, 1957-1962.

$19 . \quad$ J. R. G. Navarro, D. Manchon, F. Lerouge, E. Cottancin, J. Lerme, C. Bonnet, F. Chaput, A. Mosset, M. Pellarin and S. Parola, Nanotechnology, 2012, 23.

$8520 . \quad$ J. E. Millstone, W. Wei, M. R. Jones, H. J. Yoo and C. A. Mirkin, Nano Letters, 2008, 8, 2526-2529.

21. M. Gazda and D. W. Margerum, Inorganic Chemistry, 1994, 33, 118-123.

22. W. C. Bray and R. S. Livingston, Journal of the American $90 \quad$ Chemical Society, 1928, 50, 1654-1665.

23. F. Wang, S. Cheng, Z. H. Bao and J. F. Wang, Angewandte Chemie-International Edition, 2013, 52, 10344-10348.

$24 . \quad$ M. Z. Liu, P. Guyot-Sionnest, T. W. Lee and S. K. Gray, Physical Review B, 2007, 76, 235428.

$9525 . \quad$ T. S. Sreeprasad, A. K. Samal and T. Pradeep, Langmuir, 2007, 23, 9463-9471.

26. M. Z. Liu and P. Guyot-Sionnest, Journal of Physical Chemistry B, 2005, 109, 22192-22200.

27. J. E. Millstone, G. S. Metraux and C. A. Mirkin, Advanced

100 Functional Materials, 2006, 16, 1209-1214. 